\title{
Convergence theorems for some layout measures on random lattice and random geometric graphs *
}

\author{
Josep Díaz $\quad$ Mathew D. Penrose ${ }^{\ddagger} \quad$ Jordi Petit ${ }^{\dagger} \quad$ María Serna $^{\dagger}$
}

March 31, 1999

\begin{abstract}
This work deals with convergence theorems and bounds on the cost of several layout measures for lattice graphs, random lattice graphs and sparse random geometric graphs. For full square lattices, we give optimal layouts for the problems still open. Our convergence theorems can be viewed as an analogue of the Beardwood, Halton and Hammersley theorem for the Euclidian TSP on random points in the $d$-dimensional cube. As the considered layout measures are non-subadditive, we use percolation theory to obtain our results on random lattices and random geometric graphs. In particular, we deal with the subcritical regimes on these class of graphs.
\end{abstract}

\section{Introduction}

Layout problems on graphs aim to find a linear ordering of the nodes of an input graph such that a certain function is minimized. For the problems we consider below, finding an optimal layout is NP-hard in general, and therefore it is natural to develop and analyze techniques to obtain tight bounds on restricted instances. Graphs encoding circuits or grids are typical instances of linear arrangement problems. We consider these instances as sparse graphs that have clustering and geometric properties. For these classes of graphs, not much is known. In this paper, we are concerned with lattice graphs, random instances of lattice graphs and random geometric graphs. For most of the layout problems it is an open problem to find exact or approximated polynomial time algorithms for lattice graphs different than the full square lattice of side $n$ and with $n^{2}$ points $[9,8,7]$.

A graph is said to be a lattice graph if it is a node-induced subgraph of the infinite lattice, that is, its vertex set is a subset of $\mathbb{Z}^{2}$ and two vertices are connected whenever they are at distance one. Percolation theory provides a framework to study lattice graphs in a

\footnotetext{
${ }^{*}$ This research was partially supported by ESPRIT LTR Project no. 20244 - ALCOM-IT, CICYT Project TIC97-1475-CE, and CIRIT project 1997SGR-00366.

${ }^{\dagger}$ Departament de Llenguatges i Sistemes Informàtics. Universitat Politècnica de Catalunya. Campus Nord C6. c/ Jordi Girona 1-3. 08034 Barcelona (Spain). \{diaz,jpetit,mjserna\}@lsi.upc.es

${ }_{\ddagger}^{\ddagger}$ Department of Mathematical Sciences, University of Durham, South Road, Durham DH1 3LE, England. Mathew.Penrose@durham.ac.uk
} 
probabilistic setting. We consider site percolation, where nodes from the infinite lattice are selected with some probability $p$ (selected nodes are called "open"). Let $C_{0}$ be the set of all open nodes connected by a path of open nodes to the origin. A basic question in percolation theory is whether or not $C_{0}$ can be infinite. Let $\vartheta(p)$ denote the probability that $\left|C_{0}\right|=\infty$, and set $p_{c}=\inf \{p: \vartheta(p)>0\}$, the critical value of $p$. It is well-known that $p_{c} \in(0.5,1)[6]$. In this paper, we consider only subcritical limiting regimes $p \in\left(0, p_{c}\right)$ in which all components are almost surely finite. Results for supercritical regimes are derived in $[4,13]$. In order to deal with bounded graphs, we introduce the class of random lattice graphs with parameters $m$ and $p$ denoted by $\mathcal{L}_{m, p}$ that corresponds to the lattice graphs whose set of vertices are obtained through the random selection of each element from $\{0, \ldots, m-1\}^{2}$, chosen independently with probability $p$.

A random geometric graph $\mathcal{G}\left(\mathcal{X}_{n} ; r_{n}\right)$, with $\left(r_{n}\right)$ some chosen sequence of positive numbers, is defined by a set $\mathcal{X}_{n}$ of $n$ uniform and independently distributed points on $[0,1]^{d}$ and edges formed by joining any two different points at distance less or equal than $r_{n}$. For an infinite-volume analogue, let $\mathcal{P}_{\lambda}$ denote a homogeneous Poisson process on $\mathbb{R}^{d}$ of intensity $\lambda$, and set $\mathcal{P}_{\lambda, 0}=\mathcal{P}_{\lambda} \cup\{0\}$. For $n$ large, after appropriate scaling and centering at a randomly chosen point of $\mathcal{X}_{n}$, the graph $\mathcal{G}\left(\mathcal{X}_{n} ; r_{n}\right)$ looks locally like $\mathcal{G}\left(\mathcal{P}_{\lambda, 0} ; 1\right)$. We consider a continuum site percolation process based on the Poisson process; let $\widetilde{\vartheta}(\lambda)$ be the probability that the added point at the origin lies in an infinite component of $\mathcal{G}\left(\mathcal{P}_{\lambda, 0} ; 1\right)$. Then define the critical percolation $\lambda_{c}$ as the infimum of $\{\lambda>0: \widetilde{\vartheta}(\lambda)>0\}$. It is well known [6] that $\lambda_{c} \in(0, \infty)$. In this paper we shall deal with random geometric graphs satisfying the condition $\lim _{n \rightarrow \infty} n r_{n}^{d}=\lambda$, for the subcritical regime $\lambda<\lambda_{c}$.

Our layout problems are formally defined as follows. A layout $\varphi$ on a graph $G=(V, E)$ is a one-to-one function $\varphi: V \rightarrow\{1, \ldots, n\}$ with $n=|V|$. Given a graph $G$, a layout $\varphi$ on $G$ and a number $i<n$, let us define the sets:

$$
L(i, \varphi, G)=\{u \in V(G): \varphi(u) \leq i\} \quad \text { and } \quad R(i, \varphi, G)=\{u \in V(G): \varphi(u)>i\},
$$

the measures:

$$
\begin{aligned}
\theta(i, \varphi, G) & =|\{u v \in E(G): u \in L(i, \varphi, G) \wedge v \in R(i, \varphi, G)\}|, \\
\delta(i, \varphi, G) & =|\{u \in L(i, \varphi, G): \exists v \in R(i, \varphi, G): u v \in E(G)\}|, \\
\lambda(u v, \varphi, G) & =|\varphi(u)-\varphi(v)| \quad \text { where } u v \in E(G),
\end{aligned}
$$

and the problems:

- Minimum Linear Arrangement (MinLA): Given a graph $G=(V, E)$, find $\operatorname{MinlA}(G)=\min _{\varphi} \sum_{u v \in E} \lambda(u v, \varphi, G)=\min _{\varphi} \sum_{i=1}^{n} \theta(i, \varphi, G)$. 
- Minimum Cut Width (MinCut): Given a graph $G=(V, E)$, find $\operatorname{MinCUT}(G)=\min _{\varphi} \max _{i=1}^{n} \theta(i, \varphi, G)$.

- Vertex Separation (VertSeP): Given a graph $G=(V, E)$, find $\operatorname{MinVS}(G)=\min _{\varphi} \max _{i=1}^{n} \delta(i, \varphi, G)$.

- Minimum Sum Cut (MinSumCut): Given a graph $G=(V, E)$, find $\operatorname{MiNSC}(G)=\min _{\varphi} \sum_{i=1}^{n} \delta(i, \varphi, G)$.

- Bisection (Bisection): Given a graph $G=(V, E)$, find $\operatorname{MinBIS}(G)=\min _{\varphi} \theta(\lfloor n / 2\rfloor, \varphi, G)$.

- Vertex Bisection (MinVertBis): Given a graph $G=(V, E)$, find $\operatorname{MinvB}(G)=\min _{\varphi} \delta(\lfloor n / 2\rfloor, \varphi, G)$.

The defined problems have important applications in several different areas, see for example [4]. With regard to their complexity, MinCut and VERTSEP remain NP-complete even when restricted to lattice graphs and geometric graphs [4]. For the remaining layout problems the complexity on lattice graphs and geometric graphs is open.

In this paper, we first present optimal layouts for MINVs, MINVB, and MINSC on full square lattices. Previously, the only known optimal layouts for these graphs were for MINCUT, MINBIS and MINLA $[7,9,8]$. Results for the case of $d$-dimensional $c$-ary arrays (a generalization of square lattices) on the Bisection, MinCut and MinLA problems are presented in [10]. On the other hand, [11] presents a dynamic programming algorithm to solve BISECTION on lattice graphs without holes. Then, we consider general lattice graphs, and we present upper bounds for several layout problems on any lattice graph. Afterwards, we move to a randomized setting where we deal with random lattice and random geometric graphs. The main result for these graphs can be viewed as an analogue of the celebrated Beardwood, Halton and Hammersley theorem on the cost for the traveling salesman problem (TSP) on random points distributed in $[0,1]^{d}$,

BHH Theorem [1]. Let $X=\left\{X_{i}\right\}$ be a sequence of independent and uniformly distributed points in $[0,1]^{d}$. Let $\operatorname{mintsP}(n)$ denote the length of the optimal solution of the TSP among the first $n$ points of $X$. Then, there exists a constant $\beta(d)$ such that $\operatorname{MinTsP}(n) / n^{(d-1) / d}$ converges to $\beta(d)$ almost surely as $n \rightarrow \infty$.

A key property to prove BHH-like results is geometric subadditivity (see Chapter 3 of [14]). This property does not hold for our layout problems, therefore we take a completely different approach using percolation theory. Except for BisEction and MinVertBis, one property that all these problems share is monotonicity, that is, the optimal value on a subgraph is always less than or equal to the optimal value in the whole graph. 
$\sum_{i=3}^{2}(2-2)$

\begin{tabular}{||c|c|c|c|c|c|c|||}
\hline$n$ & $n$ & $n-1$ & $\vdots$ & 4 & 3 & 0 \\
\hline$n-1$ & $n$ & $n$ & $n-1$ & $\ddots$ & 4 & 2 \\
\hline$n$ & $n-1$ & $n$ & $n$ & $n-1$ & & 3 \\
\hline 3 & 4 & $\ddots$ & $n-1$ & $n$ & $n$ & $n-2$ \\
\hline 2 & 3 & 4 & $n-1$ & $n$ & $n-1$ \\
\hline 1 & 2 & 3 & 4 & $\vdots$ & $n-1$ \\
\hline
\end{tabular}

Figure 1: Values of the vertex cut in the diagonal ordering $\varphi_{D}$.

\section{Bounds for lattice graphs}

We begin this section by characterizing the optimal layouts for some of the problems defined in the previous section on square lattice graphs. Then we give some deterministic upper bounds on the costs of the defined problems on finite subsets of the integer lattice $\mathbb{Z}^{2}$.

Each subset $L$ of vertices in $\mathbb{Z}^{2}$ is identified with a lattice graph, namely the maximal subgraph of the 2-dimensional integer lattice with vertex set $L$. Let $L_{n}$ be the full $n \times n$ square lattice graph. The next results concern the optimality of the diagonal ordering $\varphi_{D}$ on $L_{n}$. In this ordering, $\mathbf{x}=(x, y)$ precedes $\mathbf{x}^{\prime}=\left(x^{\prime}, y^{\prime}\right)$ whenever $x+y<x^{\prime}+y^{\prime}$, and whenever $x+y=x^{\prime}+y^{\prime}$ and $x<x^{\prime}$.

Let $1 \leq k<n^{2}$ and $\mathbf{x}=(x, y)$ such that $\varphi_{D}(\mathbf{x})=k$. Define $r \in \mathbb{Z}^{+}$as follows: If $x+y \leq n$, then $r=x+y-1$, then we have $r(r-1) / 2<k \leq r(r+1) / 2$ and $\delta\left(k, \varphi_{D}, L_{n}\right)=r$. If $x+y>n$ with $x \neq n$, then $r=2 n-(x+y)+1$, and when $x=n$ then $r=2 n-(x+y)$. In this two last cases we have $r(r-1) / 2<n^{2}-k \leq r(r+1) / 2$ and $\delta\left(k, \varphi_{D}, L_{n}\right)=r+1$. In particular, for all $k, \delta\left(k, \varphi_{D}, L_{n}\right) \leq n$. See Figure 1 .

Proposition 1 (Vertex isoperimetric inequality). For any layout $\varphi$ on $L_{n}$ and any $k \in$ $\left\{1, \ldots, n^{2}\right\}$, we have $\delta\left(k, \varphi, L_{n}\right) \geq \delta\left(k, \varphi_{D}, L_{n}\right)$.

Proof. Given $\varphi$ and $k$, let $A$ be the set $L\left(k, \varphi, L_{n}\right)$, and let $\partial_{\text {in }} A$ be the number of boundary elements of $A$, i.e. elements of $A$ having neighbours in $L_{n} \backslash A$. Thus $\partial_{\text {in }} A=\delta\left(k, \varphi, L_{n}\right)$. 
Let $A^{\prime}$ be the set in $L_{n}$ obtained by "pushing each vertical section of $A$ down as far as possible towards the $x$-axis"; more precisely, setting $S_{i}(A)=\{j:(i, j) \in A\}$ for $i \in\{1, \ldots, n\}$, let

$$
A^{\prime}=\cup_{i \in\{1, \ldots, n\}: S_{i}(A) \neq \emptyset}\{i\} \times\left\{1, \ldots,\left|S_{i}(A)\right|\right\} .
$$

Notice that $\left|A^{\prime}\right|=|A|$, and it is not hard to check that $\partial_{\text {in }} A^{\prime} \leq \partial_{\text {in }} A$.

Let $A^{\prime \prime}$ be the set in $L_{n}$, obtained by "pushing each horizontal section of $A^{\prime}$ sideways as far as possible towards the $y$-axis", in an analogous manner to the construction of $A^{\prime}$ from $A$. Then $\left|A^{\prime \prime}\right|=\left|A^{\prime}\right|=|A|$, and $\partial_{\text {in }} A^{\prime \prime} \leq \partial_{\text {in }} A^{\prime} \leq \partial_{\text {in }} A$. Moreover, $A^{\prime \prime}$ is a down-set, that is, it has the property that for any $\mathbf{x} \in A^{\prime \prime}$, all vertices of $L_{n}$ lying directly below or directly to the left of $\mathbf{x}$ are in $A^{\prime \prime}$. Hence, without loss of generality, from now on we assume that $A$ is a down-set.

First suppose $(1, n) \notin A$ and $(n, 1) \notin A$. Choose the positive integer $r$ so that $r(r-$ $1) / 2<k \leq r(r+1) / 2$. Then there must be a point $\mathbf{x}=(x, y) \in A$ with $x+y \geq r+1$. Choose such a point $\mathbf{x}$, having neighbours in $L_{n} \backslash A$ both to its right and above it. Then there is a path of $y$ or more boundary points of $A$ from the bottom of the square to $\mathbf{x}$, and another path of $x$ or more boundary points of $A$ from the left of the square to $\mathbf{x}$, and these paths do not intersect each other except at $\mathbf{x}$. Therefore

$$
\delta\left(k, \varphi, L_{n}\right)=\partial_{\text {in }} A=y+x-1 \geq r .
$$

If $r \leq n$ then we have $\delta\left(k, \varphi_{D}, L_{n}\right)=r \leq \delta\left(k, \varphi, L_{n}\right)$, while if $r>n$ then we have $\delta\left(k, \varphi_{D}, L_{n}\right) \leq n \leq \delta\left(k, \varphi, L_{n}\right)$. Thus we get the inequality claimed.

Next suppose $(1, n) \in A$ and $(n, 1) \in A$. Choose the positive integer $r$ so that $r(r-$ $1) / 2<n^{2}-k \leq r(r+1) / 2$. Then there must be a point $\mathbf{x}=(x, y) \in L_{n} \backslash A$ with $(n+1-$ $x)+(n+1-y) \geq r+1$, that is, $x+y \leq 2 n+1-r$. Choose such a point $\mathbf{x}$, having neighbours in $A$ both to its left and above it. Then there is a path of at least $n-y+1$ boundary points of $A$ from the top of the square to the point just to the left of $\mathbf{x}$, and another path of at least $n-x+1$ boundary points of $A$ from the right of the square to just below $\mathbf{x}$, and these paths do not intersect. Therefore

$$
\delta\left(k, \varphi, L_{n}\right)=\partial_{\text {in }} A \geq 2 n-x-y+2 \geq r+1 .
$$

If $r<n$ then $\delta\left(k, \varphi_{D}, L_{n}\right)=r+1 \leq \delta\left(k, \varphi, L_{n}\right)$, while if $r \geq n$ then $\delta\left(k, \varphi_{D}, L_{n}\right) \leq n \leq$ $\delta\left(k, \varphi, L_{n}\right)$. Thus in this case we have the inequality claimed.

Finally, consider the case when only one of the the corners $(n, 1)$ and $(1, n)$ is in $A$. In these cases we have $\partial_{\text {in }} A=n \geq \delta\left(k, \varphi_{D}, L_{n}\right)$.

The previous Proposition is a special case of Corollary 9 in [2], who in fact prove the $d$-dimensional version for arbitrary $d$. We believe our proof for $d=2$ is of interest by itself. 
Theorem 1. For any $n, \varphi_{D}$ is optimal for the VertSep, MinSumCut and MinVertBis problems on $\left(L_{n}\right)$. Moreover, $\operatorname{Minvs}\left(L_{n}\right)=\operatorname{MinvB}\left(L_{n}\right)=n$ and $n^{-3} \operatorname{MinsC}\left(L_{n}\right) \rightarrow 2 / 3$ as $n \rightarrow \infty$.

Proof. The previous isoperimetric inequality yields the optimality of $\varphi_{D}$ for the costs of MINVS, MINSC and MINVB on $L_{n}$. Also we get that $\operatorname{minvs}\left(L_{n}\right)=\operatorname{MinVB}\left(L_{n}\right)=n$. To compute the sum of the cuts for $\varphi_{D}$, consider for each point in the lattice the value of the vertex cut produced by the diagonal ordering (see Figure 1), then arranging the sum by points with the same vertex cut, we get

$$
\operatorname{MiNSC}\left(L_{n}\right)=\sum_{i=1}^{n} \sum_{j=1}^{n} j+\sum_{i=3}^{n} i(i-2)+\sum_{i=2}^{(n-1)} i=\frac{2}{3} n^{3}+\frac{1}{2} n^{2}-\frac{7}{6} n .
$$

Lemma 1. For any lattice graph $L$ with $n$ vertices, and any $m \in\{1,2, \ldots, n\}$, there is a layout $\varphi$ on $L$ such that $\theta(m, \varphi, L) \leq 2^{3 / 2} \sqrt{n}+1$.

Proof. We are looking for a subset $S$ of $L$ consisting of $m$ vertices, such that there are at most $2^{3 / 2} \sqrt{n}+1$ edges between $S$ and $L \backslash S$.

Let $\alpha>0$ be a constant, to be chosen later. For $x \in \mathbb{Z}$ let $S_{x}=\{y \in \mathbb{Z}:(x, y) \in L\}$ and let $V=\left\{x \in \mathbb{Z}:\left|S_{x}\right| \geq \alpha \sqrt{n}\right\}$. For $i \in \mathbb{Z}$, let $H_{i}$ denote the half-space $(-\infty, i] \times \mathbb{R}$. Set

$$
i_{0}=\min \left\{i \in \mathbb{Z}:\left|L \cap H_{i}\right| \geq m\right\} .
$$

Consider the case $i_{0} \notin V$. Then define $S$ to be a set of the form

$$
S=L \cap\left(H_{i_{0}-1} \cup\left(\left\{i_{0}\right\} \times(-\infty, j]\right)\right)
$$

with $j$ chosen so that $S$ has precisely $m$ elements.

With this definition of $S$ for $i_{0} \notin V$, the number of horizontal edges between $S$ and $L \backslash S$ is at most $\left|S_{i_{0}}\right|$, and hence is at most $\alpha \sqrt{n}$. There is at most one vertical edge between $S$ and $L \backslash S$, so the number of edges from $S$ to $L \backslash S$ is at most $\alpha \sqrt{n}+1$ when $i_{0} \notin V$.

Now consider the other case $i_{0} \in V$. Let $I=\left[i_{1}, i_{2}\right]$ be the largest integer interval which includes $i_{0}$ and is contained in $V$. Then $i_{1}-1 \notin V$, and $i_{2}+1 \notin V$. Also, as $|V| \leq \alpha^{-1} \sqrt{n}$, $i_{2}-i_{1}+1 \leq \alpha^{-1} \sqrt{n}$. We have

$$
\left|L \cap H_{i_{1}-1}\right|<m \leq\left|L \cap H_{i_{2}}\right|
$$

For $j \in \mathbb{Z}$ let $T_{j}=\left[i_{1}, i_{2}\right] \times(-\infty, j]$. Choose $j_{0}$ so that

$$
\left|L \cap\left(H_{i_{1}-1} \cup T_{j_{0}-1}\right)\right|<m \leq\left|L \cap\left(H_{i_{1}-1} \cup T_{j_{0}}\right)\right|,
$$


and let $S$ be $L \cap\left(H_{i_{1}-1} \cup T_{j_{0}-1} \cup\left(\left[i_{1}, i_{3}\right] \times\left\{j_{0}\right\}\right)\right)$, with $i_{3} \in\left[i_{1}, i_{2}\right]$ chosen so that $S$ has precisely $m$ elements.

We estimate the number of edges between $S$ and $L \backslash S$ for the case $i_{0} \in V$. Since $i_{1}-1 \notin V$, and $i_{2}+1 \notin V$, the number of horizontal edges between $S$ and $L \backslash S$ is at most $2 \alpha \sqrt{n}+1$. Also, since $i_{2}-i_{1}+1 \leq \alpha^{-1} \sqrt{n}$, the number of vertical edges between $S$ and $L \backslash S$ is at most $\alpha^{-1} \sqrt{n}$. Combining these estimates we find that there are at most $\left(2 \alpha+\alpha^{-1}\right) \sqrt{n}+1$ edges between $S$ and $L \backslash S$, whether or not $i_{0} \in V$.

The minimum value of $2 \alpha+\alpha^{-1}$ (achieved at $\alpha=2^{-1 / 2}$ ) is $2 \sqrt{2}$. Setting $\alpha=2^{-1 / 2}$ in the above definition, we have the partition required.

Using Lemma 1, taking $m=\lfloor n / 2\rfloor$ and the fact that $\operatorname{MinvB}(L) \leq \operatorname{MinBIS}(L)$, we get the following result.

Theorem 2. For any lattice graph $L$ with $n$ vertices $\operatorname{MinBIS}(L) \leq 2^{3 / 2} \sqrt{n}+1$ and $\operatorname{MinvB}(L) \leq$ $2^{3 / 2} \sqrt{n}+1$.

For the MinCut problem the bound changes in the constant,

Theorem 3. For any lattice graph $L$ with $n$ vertices, $\operatorname{MinCUT}(L) \leq 14 \sqrt{n}$.

Proof. First suppose we have $n=2^{m}$ for an integer $m$. The proof is based on recursive bisection, with the cut size guaranteed by Lemma 1. Let $f(m)$ denote the maximum MINCUT cost of all lattice graphs with $2^{m}$ vertices; then $f(m)$ satisfies the following recurrence:

$$
f(m) \leq \begin{cases}0 & \text { if } m=0, \\ 2^{3 / 2} 2^{m / 2}+1+f(m-1) & \text { otherwise. }\end{cases}
$$

Then, solving the recurrence, we get

$$
f(m) \leq \sum_{j=1}^{m}\left(2^{3 / 2} 2^{j / 2}+1\right)=4\left(2^{1 / 2}+1\right)\left(2^{m / 2}-1\right)+m .
$$

We can drop the assumption that $n=2^{m}$, by taking $m$ so that $n \leq 2^{m}<2 n$, and adding extra points until one has a set of size $2^{m}$. By monotonicity this process does not reduce the MINCUT cost, so

$$
\begin{aligned}
\operatorname{MiNCUT}(L) & \leq 2^{5 / 2}\left(2^{1 / 2}+1\right) \sqrt{n}+\left(\log _{2}(n)+1\right)-4\left(2^{1 / 2}+1\right) \\
& \leq 13.657 \sqrt{n}+\log _{2}(n)-8
\end{aligned}
$$

But notice that for any $x>0$ we have $\left(\log _{2}(x)-8\right) / \sqrt{x}<0.067$; therefore the above bound for $\operatorname{MinCUT}(L)$ is at most $14 \sqrt{n}$ for all $n$. 
As a consequence of the previous theorem, and the fact that for any graph $G, \operatorname{MinLA}(G) \leq$ $n \operatorname{mincut}(G), \operatorname{Minsc}(G) \leq n \operatorname{Minvs}(G)$, and $\operatorname{minvs}(G) \leq \operatorname{Mincut}(G)$, we can extend the previous result to the remaining problems.

Corollary 1. For any lattice graph $L$ with $n$ vertices, $\operatorname{minvs}(L) \leq 14 \sqrt{n}, \operatorname{Minla}(L) \leq$ $14 n \sqrt{n}$ and $\operatorname{MinsC}(L) \leq 14 n \sqrt{n}$.

In the case of the full square lattice graphs with side $n$, the above upper bounds are within a constant of their optimal costs.

\section{Convergence results for random lattice graphs}

Let us describe some basic concepts of site percolation for the lattice $L_{m}$ with vertex set $V_{m}=([0, m) \cap \mathbb{Z})^{2}$. Given $p \in(0,1)$, site percolation with parameter $p$ on $L_{m}$ is obtained by taking a random set of open vertices of $V_{m}$ with each vertex being open with probability $p$ independently of the others. Let $\mathcal{L}_{m, p}$ be the subgraph of $L_{m}$ obtained by taking all edges between open vertices. We say that $\mathcal{L}_{m, p}$ is a random lattice graph. Denote $\mathbf{P r}_{p}$ and $\mathbf{E}_{p}$ the probability and expectation with respect to the described process of site percolation with parameter $p$. By a cluster we mean the set of vertices in any connected component of $\mathcal{L}_{m, p}$. Let $\widetilde{C}_{0}$ denote the cluster in $\mathcal{L}_{m, p}$ that includes $(0,0)$ (possibly the empty set) and let $\widetilde{C}_{x}$ denote the cluster in $\mathcal{L}_{m, p}$ that includes the point $x$.

A similar site percolation process can be generated analogously on the infinite lattice with vertex set $\mathbb{Z}^{2}$ and edges between nearest neighbors. In the same way we can extend $\mathbf{P r}_{p}$ and $\mathbf{E}_{p}$ to this infinite process. Let us denote by $C_{0}$ the cluster including the origin for site percolation on $\mathbb{Z}^{2}$. It may be the case that $C_{0}$ is empty. Notice that we can view the random lattice graph as generated by a site percolation process on $\mathbb{Z}^{2}$ and taking the open vertices in $V_{m}$.

In this section we consider random lattice graphs generated by subcritical limiting regimes $\left(p<p_{c}\right)$, in which all clusters in the infinite process are almost surely finite. We begin by giving bounds for the MiNCUT and VERTSEP problems on the subcritical percolation process on the lattice $L_{m}$.

Theorem 4. Assume $0<p<p_{c}$, there exists constants $0<c_{1}<c_{2}$ such that

$$
\lim _{m \rightarrow \infty} \operatorname{Pr}\left[c_{1} \leq \frac{\operatorname{MiNVs}\left(\mathcal{L}_{m, p}\right)}{\sqrt{\log m}} \leq \frac{\operatorname{MiNCUT}\left(\mathcal{L}_{m, p}\right)}{\sqrt{\log m}} \leq c_{2}\right]=1
$$

Proof. Recall that for any graph $G$, Minvs $(G) \leq \operatorname{MincUt}(G)$. The Mincut of a disconnected graph is the maximum of the MINCUTs of its connected components. Hence, for any positive constant $c_{2}$,

$$
\operatorname{Pr}\left[\operatorname{MinCUT}\left(\mathcal{L}_{m, p}\right) \geq c_{2} \sqrt{\log m}\right]=\operatorname{Pr}\left[\cup_{x \in V_{m}}\left\{\operatorname{MinCUT}\left(\widetilde{C}_{x}\right) \geq c_{2} \sqrt{\log m}\right\}\right]
$$


By the site percolation version of Equation 5.7 in [6], there exists $\alpha>0$ such that $\operatorname{Pr}\left[\left|C_{0}\right| \geq n\right] \leq$ $e^{-\alpha n}$. Therefore by Theorem 3

$$
\begin{aligned}
\operatorname{Pr}\left[\operatorname{MinCUt}\left(\mathcal{L}_{m, p}\right) \geq c_{2} \sqrt{\log m}\right] & \leq \operatorname{Pr}\left[\cup_{x \in V_{m}}\left\{\left|\widetilde{C}_{x}\right| \geq\left(c_{2} / 14\right)^{2} \log m\right\}\right] \\
& \leq m^{2} \exp \left(-\alpha\left(c_{2} / 14\right)^{2} \log m\right)
\end{aligned}
$$

Choosing $c_{2}>14 \sqrt{2 / \alpha}$ we get $\operatorname{Pr}\left[\operatorname{Mincut}\left(\mathcal{L}_{m, p}\right) \geq c_{2} \sqrt{\log m}\right] \rightarrow 0$.

To get a lower bound for $\operatorname{minvs}\left(\mathcal{L}_{m, p}\right)$, let $\delta>0$ and let $T_{1}, \ldots, T_{j(m)}$ be disjoint lattice subsquares of $L_{m}$, each of side $\left\lfloor(\delta \log m)^{1 / 2}\right\rfloor$, where $j(m)=\left\lfloor m /\left\lfloor(\delta \log m)^{1 / 2}\right\rfloor\right\rfloor^{2}$. Set $\gamma=\log (1 / p)$ so that $p=e^{-\gamma}$. Let $A_{j}$ be the event that all sites in $T_{j}$ are open. Then

$$
\operatorname{Pr}\left[A_{j}\right]=\exp \left(-\gamma\left\lfloor(\delta \log m)^{1 / 2}\right\rfloor^{2}\right) \geq m^{-\gamma \delta} .
$$

Hence, $\operatorname{Pr}\left[\cap_{i=1}^{j(m)} A_{i}^{c}\right] \leq\left(1-m^{-\gamma \delta}\right)^{j(m)} \leq \exp \left(-m^{-\gamma \delta} j(m)\right)$, which tends to zero provided $\delta$ is chosen so that $\gamma \delta<2$. As $\operatorname{Minvs}\left(L_{m}\right)=m$ by Theorem 1, we get

$$
\cup_{i=1}^{j(m)} A_{j} \subset\left\{\operatorname{MinVs}\left(\mathcal{L}_{m, p}\right) \geq(\delta \log m)^{1 / 2}\right\}
$$

Taking $c_{1}=\sqrt{\delta}$ we obtain the lower bound.

Notice that the above theorem only gives an order of magnitude result for the minimal cost and we do not have a convergence result. The order of magnitude is $\Theta(\sqrt{\log m})$, which contrasts with the supercritical case $p>p_{c}$, for which $\operatorname{minvs}\left(\mathcal{L}_{m, p}\right)$ and $\operatorname{MincUt}\left(\mathcal{L}_{m, p}\right)$ are $\Theta(m)[13]$.

In the next lemma we prove that for subcritical site percolation with parameter $p$, the expected ratio of the $\operatorname{MINLA}\left(C_{0}\right)$ and $\left|C_{0}\right|$ is finite. We also give a similar result for the MinSumCut problem. To cover the case $C_{0}=\emptyset$, we use the convention $0 / 0=0$, throughout the remainder of the paper.

Lemma 2. For any $p \in\left(0, p_{c}\right)$,

$$
\mathbf{E}_{p}\left[\frac{\operatorname{minL}\left(C_{0}\right)}{\left|C_{0}\right|}\right] \in(0, \infty) \text { and } \mathbf{E}_{p}\left[\frac{\operatorname{Minsc}\left(C_{0}\right)}{\left|C_{0}\right|}\right] \in(0, \infty) .
$$

Proof. Let $R_{0}=\min \left\{n: C_{0} \subset[-n, n]^{2}\right\}$; then by considering the lexicographic ordering of vertices one sees that $\operatorname{MiNSC}\left(L_{m}\right) \leq m^{3}$ and $\operatorname{MiNLA}\left(L_{m}\right) \leq m^{3}$, which together with monotonicity gives us that $\operatorname{Minsc}\left(C_{0}\right) \leq\left(2 R_{0}+1\right)^{3}$ and $\operatorname{MinLA}\left(C_{0}\right) \leq\left(2 R_{0}+1\right)^{3}$. The statement of the lemma follows from the fact that $\operatorname{Pr}_{p}\left[R_{0}>n\right]$ decays exponentially in $n$ (again, see chapter 5 of $[6]$ ).

We use this lemma to state one of our main results, namely that the value of MINLA on random lattices, divided by $m^{2}$, converges in probability to a constant. Recall (see for example 
[3]), that if $\left\{X_{n}\right\}$ is a sequence of random variables and let $X$ be a random variable, $X_{n}$ converges in probability to $X\left(X_{n} \stackrel{\operatorname{Pr}}{\longrightarrow} X\right)$ if, for every $\epsilon>0$ we have $\lim _{n \rightarrow \infty} \operatorname{Pr}\left[\left|X_{n}-X\right|>\epsilon\right]=$ 0 .

Theorem 5. Assume $0<p<p_{c}$, then as $m \rightarrow \infty$

$$
\frac{\operatorname{MiNLA}\left(\mathcal{L}_{m, p}\right)}{m^{2}} \stackrel{\operatorname{Pr}}{\longrightarrow} \mathbf{E}_{p}\left[\frac{\operatorname{MiNLA}\left(C_{0}\right)}{\left|C_{0}\right|}\right], \quad \text { and } \quad \frac{\operatorname{MinsC}\left(\mathcal{L}_{m, p}\right)}{m^{2}} \stackrel{\operatorname{Pr}}{\longrightarrow} \mathbf{E}_{p}\left[\frac{\operatorname{MinSC}\left(C_{0}\right)}{\left|C_{0}\right|}\right]
$$

Proof. Recall $\widetilde{C}_{x}$ is the cluster including $x$ for $\mathcal{L}_{m, p}$. Consider $\mathcal{L}_{m, p}$ as being embedded in a site percolation process on the infinite lattice $\mathbb{Z}^{2}$, with clusters in this latter process denoted $C_{x}$. Then,

$$
\begin{aligned}
\frac{\operatorname{MiNLA}\left(\mathcal{L}_{m, p}\right)}{m^{2}} & =m^{-2} \sum_{x \in V_{m}} \frac{\operatorname{MinLA}\left(\widetilde{C}_{x}\right)}{\left|\widetilde{C}_{x}\right|} \\
& =m^{-2} \sum_{x \in V_{m}} \frac{\operatorname{MinLA}\left(C_{x}\right)}{\left|C_{x}\right|}+m^{-2} \sum_{x \in V_{m}}\left(\frac{\operatorname{MinLA}\left(\widetilde{C}_{x}\right)}{\left|\widetilde{C}_{x}\right|}-\frac{\operatorname{MinLA}\left(C_{x}\right)}{\left|C_{x}\right|}\right) .
\end{aligned}
$$

Using Theorem VII.6.9 from [5] and the Kolmogorov zero-one law,

$$
m^{-2} \sum_{x \in V_{m}} \frac{\operatorname{MinLA}\left(C_{x}\right)}{\left|C_{x}\right|} \stackrel{\operatorname{Pr}}{\rightarrow} \mathbf{E}_{p}\left[\frac{\operatorname{MinLA}\left(C_{0}\right)}{\left|C_{0}\right|}\right] .
$$

Writing $\partial V_{m}$ for the set of $x \in V_{m}$ with lattice neighbors in $\mathbb{Z}^{2} \backslash V_{m}$, we get

$$
\begin{aligned}
m^{-2} \sum_{x \in V_{m}}\left|\frac{\operatorname{Minla}\left(\widetilde{C}_{x}\right)}{\left|\widetilde{C}_{x}\right|}-\frac{\operatorname{Minla}\left(C_{x}\right)}{\left|C_{x}\right|}\right| & \leq 2 m^{-2} \sum_{x \in V_{m}, C_{x} \neq \widetilde{C}_{x}} \frac{\operatorname{Minla}\left(C_{x}\right)}{\left|\widetilde{C}_{x}\right|} \\
& \leq 2 m^{-2} \sum_{y \in \partial V_{m}} \operatorname{Minla}\left(C_{y}\right),
\end{aligned}
$$

By the proof of Lemma $2, \mathbf{E}_{p}\left[\operatorname{MinLA}\left(C_{y}\right)\right]$ is finite and does not depend on $y$. Hence the mean of the above expression tends to zero. The result for MINLA then follows from (1), and the proof for MINSC is just the same.

\section{Convergence results for random geometric graphs}

Geometric graphs are defined as follows: Let $d \geq 2$ be an integer and let $\|\cdot\|$ be a norm on $\mathbb{R}^{d}$. Given a set $\mathcal{X} \subset \mathbb{R}^{d}$, and given $r>0$, let $\mathcal{G}(\mathcal{X} ; r)$ denote the graph with vertex set $\mathcal{X}$ and such that $x, y \in \mathcal{X}$ form an edge if and only if $\|x-y\| \leq r$ and $x \neq y$. Let $X_{1}, X_{2}, \ldots$ be independent and uniformly distributed on $[0,1]^{d}$, and let $\mathcal{X}_{n}$ be the point process $\left\{X_{1}, X_{2}, \ldots, X_{n}\right\}$. The continuum percolation probability $\widetilde{\vartheta}(\lambda)$ is the probability that the added point at the origin lies in an infinite component of $\mathcal{G}\left(\mathcal{P}_{\lambda, 0} ; 1\right)$. Set $\lambda_{c}=\inf \{\lambda>0: \widetilde{\vartheta}(\lambda)>0\}$. We deal with random geometric graphs satisfying the condition $\lim _{n \rightarrow \infty} n r_{n}^{d}=\lambda$, for $\lambda$ in the subcritical 
regime. Under the probability measure $\mathbf{P r}_{\lambda}$ with corresponding expectation $\mathbf{E}_{\lambda}$, let $C_{0}$ be the component of $\mathcal{G}\left(\mathcal{P}_{\lambda, 0} ; 1\right)$ which includes the origin.

First we deal with the behaviour of the BISEction problem. Let $\lambda_{c}^{\prime}=\inf \{\lambda>0$ : $\widetilde{\vartheta}(\lambda) \geq 1 / 2\}$; the subcritical regime for BISECTION is given by $\lambda<\lambda_{c}^{\prime}$.

Theorem 6. Suppose $\lim _{n \rightarrow \infty} n r_{n}^{d}=\lambda \in\left(0, \lambda_{c}^{\prime}\right)$. Then as $n \rightarrow \infty$,

$$
\operatorname{MiNBIS}\left(\mathcal{G}\left(\mathcal{X}_{n} ; r_{n}\right)\right) \stackrel{\operatorname{Pr}}{\longrightarrow} 0 \quad \text { and } \quad \operatorname{MINVB}\left(\mathcal{G}\left(\mathcal{X}_{n} ; r_{n}\right)\right) \stackrel{\operatorname{Pr}}{\longrightarrow} 0
$$

Proof. We need to show that with high probability, there is a subset $W$ of $\mathcal{X}_{n}$, of cardinality $\left\lfloor\frac{n}{2}\right\rfloor$, with no edges between $W$ and $\mathcal{X}_{n} \backslash W$. Recall that by hypothesis $\widetilde{\vartheta}(\lambda)<1 / 2$.

For $k \in \mathbb{N}$, set $\pi_{k}=\operatorname{Pr}_{\lambda}\left[\left|C_{0}\right|=k\right]$, and note $\pi_{k}>0$. Let $N_{n}(k)$ denote the number of points of $G\left(\mathcal{X}_{n} ; r_{n}\right)$ lying in clusters of size $k$.

Let $p_{k}(x)$ denote the probability that when adding a point $x$ to a set of $n-1$ uniformly distributed points, the new point will be in a cluster of size $k$. Then,

$$
\mathbf{E}\left[N_{n}(k)\right]=n \int_{[0,1]^{d}} p_{k}(x) d x .
$$

For $x$ not on the boundary of $[0,1]^{d}$, we have that $p_{k}(x) \rightarrow \pi_{k}$, so by the dominated convergence theorem, $n^{-1} \mathbf{E}\left[N_{n}(k)\right] \rightarrow \pi_{k}$. To look at the variance, notice that since $N_{n}(k)\left(N_{n}(k)-\right.$ 1 ) is twice the number of pairs of points both in clusters of size $k$, if we denote by $p_{k, k}(x, y)$ the probability that when inserting points at $x$ and $y$ into a set of $n-2$ uniform points they will both be in a cluster of size $k$, then

$$
\mathbf{E}\left[N_{n}(k)\left(N_{n}(k)-1\right)\right]=n(n-1) \int_{[0,1]^{d}} \int_{[0,1]^{d}} p_{k, k}(x, y) d x d y .
$$

For points $x$ and $y$ not on the boundary with $x \neq y$, we have that $p_{k, k}(x, y) \rightarrow$ $\left(\pi_{k}\right)^{2}$, hence using again the dominated convergence theorem $\mathbf{E}\left[\left(N_{n}(k) / n\right)^{2}\right] \rightarrow \pi_{k}^{2}$. So $\operatorname{Var}\left[N_{n}(k) / n\right] \rightarrow 0$, and by Chebyshev's inequality we can conclude

$$
n^{-1} N_{n}(k) \stackrel{\operatorname{Pr}}{\longrightarrow} \pi_{k}
$$

As $1-\sum_{k} \pi_{k}=\widetilde{\vartheta}(\lambda)<1 / 2$, we can choose $k_{1}$ such that $\sum_{k \leq k_{1}} \pi_{k}>1 / 2$. This inequality together with (2) implies that with probability tending to 1 as $n$ tends to infinity,

$$
\sum_{k>k_{1}} N_{n}(k)<\left\lfloor\frac{n}{2}\right\rfloor
$$

and $N_{n}(k)$ are non-zero for $k=1,2, \ldots, k_{1}$.

We generate a subset $W$ of $\mathcal{X}_{n}$ as follows. First take the union of all clusters of size greater than $k_{1}$. Then add clusters of size $k_{1}$ until there are none left. Then add clusters of 
size $k_{1}-1$ until there are none left. Continue in this way. At some point, having just added a set of size $i$, we will have a set of size $\left\lfloor\frac{n}{2}\right\rfloor-m$ with $0 \leq m<i$. If $m=0$, stop. If $m>0$ then add a cluster of size $m$ and stop. This gives a set $W \subset \mathcal{X}_{n}$, of size $\left\lfloor\frac{n}{2}\right\rfloor$, with no edges connecting $W$ to $\mathcal{X}_{n} \backslash W$, as desired.

Analogous results to those in the previous Theorem also hold for a percolation process in the lattice with $p<p_{c}^{\prime}$, defined in the same way as $\lambda_{c}^{\prime}$. Next we shall prove that in the subcritical case, the expected values of MINLA and MINSC on the induced graph on $C_{0}$ are finite.

Proposition 2. Let $\lambda<\lambda_{c}$. Then

$$
\mathbf{E}_{\lambda}\left[\operatorname{Minl} A\left(C_{0}\right)\right] \in(0, \infty) \quad \text { and } \quad \mathbf{E}_{\lambda}\left[\operatorname{Minsc}\left(C_{0}\right)\right] \in(0, \infty)
$$

Proof. Recall that for any graph $G$ with $n$ nodes, $\operatorname{minla}(G) \leq n^{3}$ and $\operatorname{Minsc}(G) \leq n^{3}$. Hence to prove the statement it is enough to show that $\mathbf{E}_{\lambda}\left[\left|C_{0}\right|^{3}\right]<\infty$. To show this, let $B(r)$ be the ball of radius $r$ centered at the origin and let

$$
\mathcal{P}_{\lambda, 0}(B(r))=\mid\left\{x \text { of } \mathcal{P}_{\lambda, 0} \mid x \in B(r)\right\} \mid
$$

Then for any $m>0$, the event $\left\{\left|C_{0}\right| \geq m^{1 / 3}\right\}$ is contained in the union of the events $\left\{\mathcal{P}_{\lambda, 0}\left(B\left(m^{1 /(6 d))} \geq m^{1 / 3}\right\}\right.\right.$ and $\left\{\operatorname{diam}\left(C_{0}\right) \geq m^{1 /(6 d)}\right\}$, therefore using Boole's inequality we get

$$
\operatorname{Pr}\left[\left|C_{0}\right| \geq m^{1 / 3}\right] \leq \operatorname{Pr}\left[\mathcal{P}_{\lambda, 0}\left(B\left(m^{1 /(6 d)}\right)\right) \geq m^{1 / 3}\right]+\operatorname{Pr}\left[\operatorname{diam}\left(C_{0}\right) \geq m^{1 /(6 d)}\right] .
$$

The first term in the right hand side is summable in $m$ by standard estimates of the Poisson distribution. The second term is summable in $m$ by Lemma 2 in [12]. Hence

$$
\sum_{m \geq 1} \operatorname{Pr}\left[\left|C_{0}\right|^{3} \geq m\right]<\infty
$$

and the statement follows.

Next we prove a technical lemma that will be needed later.

Lemma 3. The functions $\lambda \mapsto \mathbf{E}_{\lambda}\left[\frac{\operatorname{minsc}\left(C_{0}\right)}{\left|C_{0}\right|}\right]$ and $\lambda \mapsto \mathbf{E}_{\lambda}\left[\frac{\operatorname{minLA}\left(C_{0}\right)}{\left|C_{0}\right|}\right]$ are continuous in $\lambda$ on $\left(0, \lambda_{c}\right)$.

Proof. We give the proof for the MINLA case, the proof for the MINSC is similar. Define coupled versions of the Poisson process $\mathcal{P}_{\lambda}, \lambda>0$, in the following standard way. Let $\mathcal{P}$ be a Poisson process on $\mathbb{R}^{d} \times[0, \infty)$ of rate 1 , and let $\mathcal{P}_{\lambda}$ consist of the projections onto the 
first $d$ coordinates of the points of $\mathcal{P} \cap\left(\mathbb{R}^{d} \times[0, \lambda]\right)$. Using this coupling, write $C_{0}(\lambda)$ for the component including the origin of $C\left(\mathcal{P}_{\lambda} \cup\{0\} ; 1\right)$.

Suppose $\left(\lambda_{n}\right)$ is a sequence with $\lambda_{n} \rightarrow \lambda \in\left(0, \lambda_{c}\right)$. With this coupling, with probability one it is the case that for all large enough $n$ the components $C_{0}\left(\lambda_{n}\right)$ and $C_{0}(\lambda)$ are identical. Hence by the dominated convergence theorem,

$$
\mathbf{E}_{\lambda_{n}}\left[\operatorname{MinLA}\left(C_{0}\left(\lambda_{n}\right)\right) /\left|C_{0}\left(\lambda_{n}\right)\right|\right] \rightarrow \mathbf{E}_{\lambda}\left[\operatorname{MinLA}\left(C_{0}(\lambda)\right) /\left|C_{0}(\lambda)\right|\right]
$$

We give asymptotics for the MINLA and MINSC costs of the graphs $\mathcal{G}\left(\mathcal{P}_{\lambda} \cap B_{m} ; 1\right)$, where $B_{m}$ denotes the box $[0, m)^{d}$, with $m \in \mathbb{N}$.

Proposition 3. Suppose $\lambda<\lambda_{c}$, and let $\mathcal{G}_{m}=\mathcal{G}\left(\mathcal{P}_{\lambda} \cap B_{m} ; 1\right)$. Then as $m \rightarrow \infty$,

$$
\frac{\operatorname{MinLA}\left(\mathcal{G}_{m}\right)}{m^{d}} \stackrel{\operatorname{Pr}}{\rightarrow} \lambda \mathbf{E}_{\lambda}\left[\frac{\operatorname{MinLA}\left(C_{0}\right)}{\left|C_{0}\right|}\right] \text { and } \frac{\operatorname{Minsc}\left(\mathcal{G}_{m}\right)}{m^{d}} \stackrel{\operatorname{Pr}}{\longrightarrow} \lambda \mathbf{E}_{\lambda}\left[\frac{\operatorname{Minsc}\left(C_{0}\right)}{\left|C_{0}\right|}\right]
$$

Proof. We sketch a proof for MINLA. For each point $x$ of $\mathcal{P}_{\lambda} \cap B_{m}$, let $C_{x}$ denote the component of $\mathcal{G}_{m, p}\left(\mathcal{P}_{\lambda} \cap B_{m} ; 1\right)$ that includes the point $x$, and let $\widetilde{C}_{x}$ denote the component of $\mathcal{G}_{m, p}\left(\mathcal{P}_{\lambda} ; 1\right)$ that includes the point $x$. By a similar argument to the proof of Theorem 5 , it suffices to prove that

$$
\mathbf{E}_{\lambda}\left[m^{-d} \sum_{x \in \mathcal{P}_{\lambda} \cap B_{m}}\left|\frac{\operatorname{MinLA}\left(\widetilde{C}_{x}\right)}{\left|\widetilde{C}_{x}\right|}-\frac{\operatorname{MinLA}\left(C_{x}\right)}{\left|C_{x}\right|}\right|\right] \rightarrow 0 .
$$

For $l>0$, let $\partial_{l} B_{m}$ be the set of points $z \in B_{m}$ with $\|z-y\|_{\infty} \leq l$ for some $y \notin B_{m}$. The quantity inside the sum in $(3)$ is at most $\operatorname{MINLA}\left(\widetilde{C}_{x}\right) \cdot\left(C_{x} \neq \widetilde{C}_{x}\right)$, where for any statment $S$, $(S)$ stands for 1 if $S$ is true, 0 otherwise. Hence the random variable inside the expectation in $(3)$ is at most

$$
\left(m^{-d} \sum_{x \in \mathcal{P}_{\lambda} \cap \partial_{l} B_{m}} \operatorname{MiNLA}\left(\widetilde{C}_{x}\right)\right)+\left(m^{-d} \sum_{x \in B_{m} \backslash \partial_{l} B_{m}} \operatorname{MinLA}\left(\widetilde{C}_{x}\right) \cdot\left(\operatorname{diam}\left(\widetilde{C}_{x}\right)>l\right)\right) .
$$

The expectation of the first term tends to zero, while the expectation of the second term equals $\lambda \mathbf{E}_{\lambda}\left[\operatorname{MinLA}\left(C_{0}\right)\left(\left|C_{0}\right|>l\right)\right]$, which can be made arbitrarily small by the choice of $l$. Then (3) follows.

Theorem 7. Suppose $\lim _{n \rightarrow \infty} n r_{n}^{d}=\lambda \in\left(0, \lambda_{c}\right)$. Then, as $n \rightarrow \infty$

$$
n^{-1} \operatorname{MinLA}\left(\mathcal{G}\left(\mathcal{X}_{n} ; r_{n}\right)\right) \stackrel{\mathbf{P r}}{\rightarrow} \mathbf{E}_{\lambda}\left[\frac{\operatorname{MiNLA}\left(C_{0}\right)}{\left|C_{0}\right|}\right] \text {, }
$$

and

$$
n^{-1} \operatorname{MinSC}\left(\mathcal{G}\left(\mathcal{X}_{n} ; r_{n}\right)\right) \stackrel{\operatorname{Pr}}{\rightarrow} \mathbf{E}_{\lambda}\left[\frac{\operatorname{MiNSC}\left(C_{0}\right)}{\left|C_{0}\right|}\right] .
$$

Moreover, both of the above limits are finite and strictly positive. 
Proof. Consider the graph $\mathcal{G}\left(\mathcal{X}_{n} ; r_{n}\right)$ with $n r_{n}^{d} \rightarrow \lambda \in\left(0, \lambda_{c}\right)$. We couple $\mathcal{X}_{n}$ to two Poisson processes with a slightly higher or lower density of points, as follows: Take $\lambda_{1}<\lambda<\lambda_{2}<\lambda_{c}$ and set $m_{n}=\left\lceil r_{n}^{-1}\right\rceil, m_{n}^{\prime}=\left\lfloor r_{n}^{-1}\right\rfloor$. Let $M_{n}$ and $M_{n}^{\prime}$ be Poisson variables with mean $\lambda_{1} m_{n}^{d}$ and $\lambda_{2}\left(m_{n}^{\prime}\right)^{d}$ respectively, independent of $\left\{X_{1}, \ldots, X_{n}\right\}$. Then, as $n \rightarrow \infty$

$$
\operatorname{Pr}\left[M_{n}>n\right] \rightarrow 0, \text { and } \operatorname{Pr}\left[M_{n}^{\prime}<n\right] \rightarrow 0
$$

Let us set $m_{n} \mathcal{X}_{n}=\left\{m_{n} X_{i}: 1 \leq i \leq n\right\}, \mathcal{P}_{n}=\left\{m_{n} X_{i}: 1 \leq i \leq M_{n}\right\}$, and $\mathcal{P}_{n}^{\prime}=$ $\left\{m_{n}^{\prime} X_{i}: 1 \leq i \leq M_{n}^{\prime}\right\}$. Notice that $\mathcal{P}_{n}$ is a Poisson processes on $B_{m_{n}}$ with intensity $\lambda_{1}$ and $\mathcal{P}_{n}^{\prime}$ is a Poisson processes on $B_{m_{n}^{\prime}}$ with intensity $\lambda_{2}$.

If $M_{n} \leq n$ then $\mathcal{G}\left(\mathcal{P}_{n} ; 1\right)$ is a subgraph of $\mathcal{G}\left(m_{n} \mathcal{X}_{n} ; m_{n} r_{n}\right)$, which is isomorphic to $\mathcal{G}\left(\mathcal{X}_{n} ; r_{n}\right)$. Similarly, if $M_{n}^{\prime} \geq n$, then $\mathcal{G}\left(m_{n}^{\prime} \mathcal{X}_{n} ; m_{n}^{\prime} r_{n}\right)$ is a subgraph of $\mathcal{G}\left(\mathcal{P}_{n}^{\prime} ; 1\right)$. By monotonicity,

$$
\operatorname{Pr}\left[\operatorname{MinLA}\left(\mathcal{G}\left(\mathcal{P}_{n} ; 1\right)\right) \leq \operatorname{MinLA}\left(\mathcal{G}\left(\mathcal{X}_{n} ; r_{n}\right)\right) \leq \operatorname{MinLA}\left(\mathcal{G}\left(\mathcal{P}_{n}^{\prime} ; 1\right)\right)\right] \rightarrow 1
$$

By Proposition 3,

$$
\frac{\operatorname{MiNLA}\left(\mathcal{G}\left(\mathcal{P}_{n} ; 1\right)\right)}{m_{n}^{d}} \stackrel{\operatorname{Pr}}{\longrightarrow} \lambda_{1} \mathbf{E}_{\lambda_{1}}\left[\frac{\operatorname{MinLA}\left(C_{0}\right)}{\left|C_{0}\right|}\right],
$$

so that

$$
\frac{\operatorname{MiNLA}\left(\mathcal{G}\left(\mathcal{P}_{n} ; 1\right)\right)}{n} \stackrel{\operatorname{Pr}}{\longrightarrow}\left(\frac{\lambda_{1}}{\lambda}\right) \mathbf{E}_{\lambda_{1}}\left[\frac{\operatorname{MinLA}\left(C_{0}\right)}{\left|C_{0}\right|}\right]
$$

Similarly,

$$
\frac{\operatorname{MiNLA}\left(\mathcal{G}\left(\mathcal{P}_{n}^{\prime} ; 1\right)\right)}{n} \stackrel{\operatorname{Pr}}{\longrightarrow}\left(\frac{\lambda_{2}}{\lambda}\right) \mathbf{E}_{\lambda_{2}}\left[\frac{\operatorname{MinLA}\left(C_{0}\right)}{\left|C_{0}\right|}\right] .
$$

Taking $\lambda_{1} \uparrow \lambda$ and $\lambda_{2} \downarrow \lambda$ and using Lemma 3,

$$
\frac{\operatorname{MiNLA}\left(\mathcal{G}\left(\mathcal{X}_{n} ; r_{n}\right)\right)}{n} \stackrel{\operatorname{Pr}}{\rightarrow} \mathbf{E}_{\lambda}\left[\frac{\operatorname{MiNLA}\left(C_{0}\right)}{\left|C_{0}\right|}\right] .
$$

The proof for the convergence of MINSC is analogous.

\section{Conclusions}

In this paper we have considered several layout problems for specific classes of sparse graphs: lattice graphs, random lattice graphs and random geometric graphs. Table 1 summarizes the results.

In the case of lattice graphs, our results are given for the 2-dimensional lattice; it remains open to extend them to any dimension. The main result for random lattices is the convergence in probability to a constant of the values of $\operatorname{MinLA}\left(\mathcal{L}_{m, p}\right) / m^{2}$ and $\operatorname{MinsC}\left(\mathcal{L}_{m, p}\right) / m^{2}$. Our results on the subcritical regime together with the results obtained for the supercritical regime [13] make explicit a phase transition at $p_{c}$. An open problem is to find good methods for evaluating numerically the constants in Theorem 5 as functions of the open vertex density 
$p$, and the analogous constants in Theorem 7. Preliminary estimations for those constants were given in [4]; the used method was a raw simulation of the percolation process on the lattice and computation of lower bounds and upper bounds with heuristics.

In the last part of the paper, we have presented convergence theorems for MINLA and MINSC on random geometric graphs in the subcritical regime. For the MinLA and MinSumCut problems on random geometric graphs, there is a phase transition at $\lambda=\lambda_{c}$. Indeed, in [13] it is shown that if $\lambda>\lambda_{c}$ then $\operatorname{MinLA}\left(\mathcal{G}\left(\mathcal{X}_{n} ; r_{n}\right)\right)$ and $\operatorname{Minsc}\left(\mathcal{G}\left(\mathcal{X}_{n} ; r_{n}\right)\right)$ are $\Theta\left(n^{2-1 / d}\right)$. Our results show that the behaviour for $\lambda<\lambda_{c}$ is entirely different. For the Bisection and the MinVERTBis problems, the phase transition occurs not at $\lambda_{c}$ but at $\lambda_{c}^{\prime}$ defined by

$$
\lambda_{c}^{\prime}=\inf \{\lambda>0: \theta(\lambda) \geq 1 / 2\},
$$

and the subcritical regime for MINBIS and MINVB is given by $\lambda<\lambda_{c}^{\prime}$.

For the sake of clarity, we contented ourselves in this paper with demonstrating convergence in probability; however, the convergence in our theorems actually holds in the stronger sense of complete convergence which implies convergence almost surely (see [15]). 


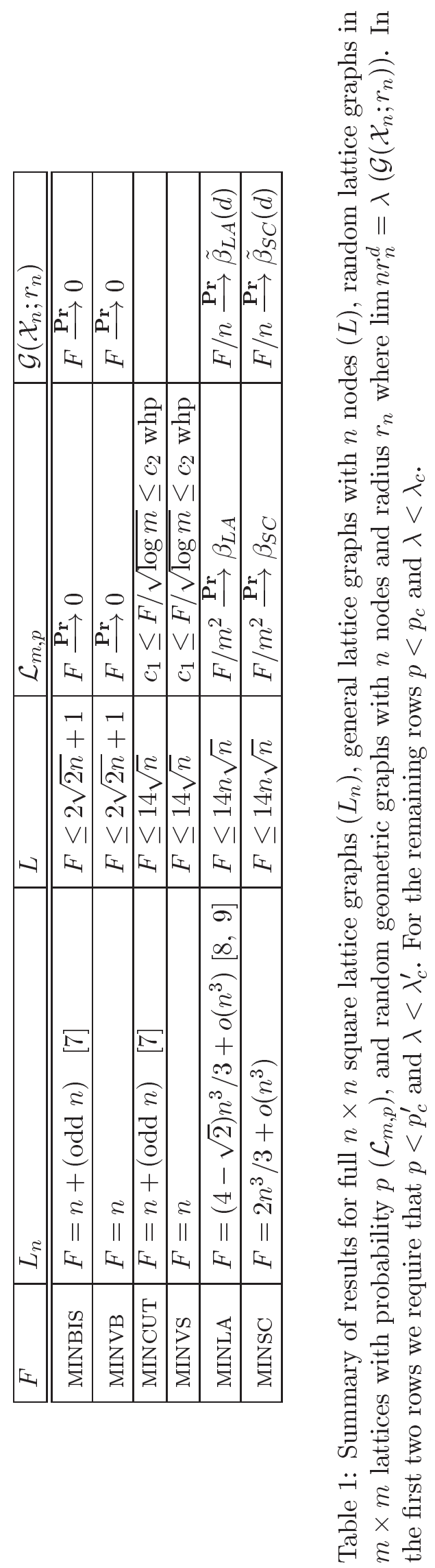




\section{References}

[1] J. Beardwood, J. Halton, and J.M. Hammersley. The shortest path through many points. Proceedings of the Cambridge Philos. Society., 55:299-327, 1959.

[2] B. Bollobas and I. Leader. Compressions and isoperimetric inequalities. Journal of Combinatorial Theory Series A, 56:47-62, 1991.

[3] K.L. Chung. A Course in Probability Theory. Academic Press, New York, 1974.

[4] J. Díaz, M. D. Penrose, J. Petit, and M. Serna. Linear orderings of random geometric graphs. Technical report, Departament de Llenguatges i Sistemes Informàtics, UPC, http://www.Isi.upc.es/ jpetit/Publications, 1999.

[5] N. Dunford and J. Schwartz. Linear Operators. Part I: General Theory. Interscience Publisher., New York, 1958.

[6] G. Grimmett. Percolation. (2nd edition). Springer-Verlag, Heidelberg, 1999.

[7] F.T. Leighton. Introduction to Parallel Algorithms and Architectures: Arrays, Trees, Hypercubes. Morgan Kaufmann, San Mateo, CA., 1993.

[8] G. Mitchison and R. Durbin. Optimal numberings of an $n \times n$ array. SIAM Journal on Discrete Mathematics, 7(4):571-582, 1986.

[9] D.O. Muradyan and T.E. Piliposjan. Minimal numberings of vertices of a rectangular lattice. Akad. Nauk. Armjan. SRR, 1(70):21-27, 1980. In Russian.

[10] K. Nakano. Linear layouts of generalized hypercubes. In J. van Leewen, editor, Graphtheoretic concepts in computer science, volume 790 of Lecture Notes in Computer Science, pages 364-375. Springer-Verlag, 1993.

[11] C. H. Papadimitriou and M. Sideri. The bisection width of grid graphs. In First ACMSIAM Symp. on Discrete Algorithms., pages 405-410, San Francisco, 1990.

[12] M. Penrose. Single linkage clustering and continuum percolation. Journal of Multivariate Analysis., 53:94-109, 1995.

[13] M.D. Penrose. Vertex ordering and partition problems for random spatial graphs. Technical report, University of Durham, 1999.

[14] J.M. Steele. Probability theory and Combinatorial Optimization. SIAM CBMS-NSF Regional Conference Series in Applied Mathematics., 1997. 
[15] J.E. Yukich. Probability Theory of classical Euclidian Optimization Problems. SpringerVerlag, Heidelberg, 1998. 